Research

Open Access

\title{
Using Medical Emergency Teams to detect preventable adverse events
}

\author{
Akshai lyengar ${ }^{1}$, Alan Baxter ${ }^{2}$ and Alan J Forster ${ }^{1,3}$ \\ 1Department of Medicine, Faculty of Medicine, University of Ottawa, 451 Smyth Road, Ottawa, ON, K1H 8M5, Canada \\ ${ }^{2}$ Department of Anaesthesia, Faculty of Medicine, University of Ottawa, 451 Smyth Road, Ottawa, ON, K1H 8M5, Canada \\ ${ }^{3}$ Clinical Epidemiology Program, Ottawa Hospital Research Institute, 725 Parkdale Avenue, Ottawa, ON, K1Y 4E9, Canada
}

Corresponding author: Alan J Forster, aforster@ohri.ca

Received: 12 Feb 2009 Revisions requested: 17 Apr 2009 Revisions received: 10 Jun 2009 Accepted: 30 Jul 2009 Published: 30 Jul 2009

Critical Care 2009, 13:R126 (doi:10.1186/cc7983)

This article is online at: http://ccforum.com/content/13/4/R126

(C) 2009 lyengar et al.; licensee BioMed Central Ltd.

This is an open access article distributed under the terms of the Creative Commons Attribution License (http://creativecommons.org/licenses/by/2.0), which permits unrestricted use, distribution, and reproduction in any medium, provided the original work is properly cited.

\begin{abstract}
Introduction Medical Emergency Teams (METs), also known as Rapid Response Teams, are recommended as a patient safety measure. A potential benefit of implementing an MET is the capacity to systematically assess preventable adverse events, which are defined as poor outcomes caused by errors or system design flaws. We describe how we used MET calls to systematically identify preventable adverse events in an academic tertiary care hospital, and describe our surveillance results.
\end{abstract}

Methods For four weeks we collected standard information on consecutive MET calls. Within a week of the MET call, a multidisciplinary team reviewed the information and rated the cause of the outcome using a previously developed rating scale. We classified the type and severity of the preventable adverse event.
Results We captured information on all 65 MET calls occurring during the study period. Of these, 16 (24\%, 95\% confidence interval $[\mathrm{Cl}] 16 \%-36 \%)$ were felt to be preventable adverse events. The most common cause of the preventable adverse events was error in providing appropriate therapy despite an accurate diagnosis. One service accounted for a disproportionate number of preventable adverse events $(n=5$, [31\%, 95\% Cl 14\%-56\%]).

Conclusions Our method of reviewing MET calls was easy to implement and yielded important results. Hospitals maintaining an MET can use our method as a preventable adverse event detection system at little additional cost.

\section{Introduction}

Medical Emergency Teams (METs), alternatively known as Rapid Response Teams, have recently been implemented in many hospitals worldwide [1]. The primary role of an MET is to improve the early identification and management of acutely deteriorating ward patients [1]. Several studies demonstrate an association between MET implementation and improved hospital outcomes [2-5], although there are also negative trials [6-8]. Despite the conflicting evidence, many institutions and health systems have continued to fund MET implementations due to perceived benefits extending beyond those evaluated in the published research [9-11]. These include improvements in patient safety culture and nursing work environment.

In this study, we report on our experience with expanding the role of our institution's MET to support the detection of pre- ventable adverse events, which are defined as poor outcomes caused by medical error. We felt a systematic evaluation of patient care immediately prior to MET notification might provide useful information for system improvement because the MET is responding to critical situations in which there is at least some likelihood of prior inappropriate treatment [12-16]. Our method is a modification of a prior attempt to achieve a similar objective [17]. Our approach differs in that we wished to incorporate the evaluation as part of the routine followed by the MET during a call. We hoped that this would minimize the resources required for the task and enhance timeliness of our detection while at the same time yield useful information.

Cl: confidence interval; MET: Medical Emergency Team. 


\section{Materials and methods Setting}

The study was approved by the Ottawa Hospital Research Ethics Board. The Ottawa Hospital General Campus is a 487bed tertiary care teaching hospital. It implemented an MET in January 2005. The team is composed of a physician (intensivist during the day and a critical care resident at night), a critical care nurse, and a critical care-trained respiratory therapist. The MET can be activated by any hospital staff and is active 24 hours a day. Providers in our hospital use standard criteria for activating the MET. The MET has over 40 calls per 1,000 hospital admissions, and more than $70 \%$ of intensive care unit admissions are preceded by an MET call.

\section{Data collection}

For a 4-week period in 2007, we used a standard form to collect information on each MET call (Appendix 1 of Additional data file 1). For each MET call, we described the reason for the call, the admitting service and diagnosis, the admission status, the current acute and chronic medical conditions, a summary of the patient's hospital stay and course in hospital, the presumed explanation for the patient's deterioration, the treatment provided by the attending team prior to the MET call, the MET's treatment, and the patient's eventual outcome. The MET physician recorded data at the time of the MET call Monday through Friday during working hours. For MET calls at other times, the MET physician interviewed the providers involved in the case and reviewed the medical record. It took approximately 5 minutes to complete the form.

\section{Outcomes}

We used standard patient safety definitions [18]. An adverse outcome is any suboptimal outcome experienced by the patient. By definition, any MET call is an adverse outcome. An adverse event is an adverse outcome caused by the processes of medical management rather than by the progression of disease. Medical management refers to all aspects of care. A preventable adverse event is an adverse event caused by error or health system flaw. An error is a failure to achieve a desired objective through the failure to execute a plan correctly, through the implementation of an incorrect plan, or through omission.

\section{Case classification}

All cases were reviewed and classified by three physicians an internist (AJF), an anesthetist/intensivist (AB), and a PGY2 (post-graduate year two) internal medicine resident (Al) within 1 week of each MET call. The group of three physicians achieved consensus on whether the outcome was a result of medical management using a previously derived and widely accepted review process [19-24]. If so, the case was considered an adverse event, in which case it was further classified in terms of its preventability. Preventable adverse events were further classified as to their subtype.

\section{Consent}

We did not obtain patient or provider consent as part of the protocol. We argued successfully to our Research Ethics Board that the protocol posed minimal risk to patients or providers. The principal ethical concern was the potential of an inappropriate disclosure of personal health information. We created a case report form that did not contain usual patient or provider identifiers. Individuals could be identified only if someone obtained our case report forms and used our hospital information systems inappropriately.

\section{Statistical analysis}

We created descriptive statistics for all studied factors. We compared the distribution of these factors by preventable adverse event status using the chi-square statistic for categorical variables and the Wilcoxon rank-sum test for continuous variables. As only one variable was significantly associated with adverse event status, we did not perform a multi-variable analysis. We used SAS version 9.1 (SAS Institute Inc., Cary, NC, USA) for all analyses.

\section{Results}

Sixty-five MET calls occurred during the study period (Table 1). Patients were elderly (median age 71 years, interquartile range 60 to 82 years). Most hospital services had at least one MET call. Ninety-one percent of patients were considered 'acute care' at the time of the MET call and had been in hospital for a median of 4 days (interquartile range 2 to 12.5 days) before the call. Of the 65 calls received, 23 were considered to be adverse events (35\%, 95\% confidence interval [Cl] 25\% to $48 \%$ ) and 16 were considered to be preventable adverse events $(24 \%, 95 \% \mathrm{Cl} 16 \%$ to $36 \%)$. Calls of three of the sixteen patients with preventable adverse events were considered life-threatening (19\%, 95\% Cl 7\% to 43\%). Six MET cases and their ratings are described as examples in the text box of Additional data file 2. We describe all adverse events in Appendix 2 of Additional data file 3.

'Therapeutic errors', defined as a failure to apply the appropriate treatment regimen, contributed to the outcome in 14 of the 16 patients with preventable adverse events $(88 \%, 95 \% \mathrm{Cl}$ $64 \%$ to $97 \%$ ). The other two preventable adverse events were considered adverse drug events.

We assessed factors associated with preventable adverse event classifications (Table 1). The only characteristic associated with preventable adverse event occurrence was hospital service. Service $\mathrm{C}$ was noted to have a high proportion of calls related to preventable adverse events. Although service $A$ accounted for the most calls, it accounted for only one preventable adverse event. All other studied factors were not associated with preventable adverse event status. 
Table 1

\begin{tabular}{|c|c|c|c|c|}
\hline Characteristic & All & Patients with preventable AEs & Patients without preventable AEs & $P$ value \\
\hline Number & 65 & 16 & 49 & N/A \\
\hline Age, years & $71(60-81)$ & $76(68-82)$ & $68(59-81)$ & 0.32 \\
\hline Service & & & & 0.02 \\
\hline A & $12(18 \%)$ & $1(6 \%)$ & $11(22 \%)$ & \\
\hline B & $8(12 \%)$ & $2(13 \%)$ & $6(12 \%)$ & \\
\hline $\mathrm{C}$ & $7(11 \%)$ & $5(31 \%)$ & $2(4 \%)$ & \\
\hline $\mathrm{D}$ & $5(8 \%)$ & $2(13 \%)$ & $3(6 \%)$ & \\
\hline$E$ & $5(8 \%)$ & $1(6 \%)$ & $4(8 \%)$ & \\
\hline $\mathrm{F}$ & $4(6 \%)$ & $2(13 \%)$ & $2(4 \%)$ & \\
\hline Other & $24(37 \%)$ & $3(19 \%)$ & $21(43 \%)$ & \\
\hline Admission status & & & & 0.31 \\
\hline Acute & $59(91 \%)$ & $14(88 \%)$ & $45(92 \%)$ & \\
\hline Chronic & $6(9 \%)$ & $2(13 \%)$ & $4(8 \%)$ & \\
\hline Length of stay, days ${ }^{a}$ & $4(2-12.5)$ & $4(3-21)$ & $4(2-11)$ & 0.51 \\
\hline Call indication & & & & 0.23 \\
\hline Blood pressure & $23(35 \%)$ & $5(31 \%)$ & $18(37 \%)$ & \\
\hline Airway & $11(17 \%)$ & $1(6 \%)$ & $10(20 \%)$ & \\
\hline Heart rate & $8(12 \%)$ & $3(19 \%)$ & $5(10 \%)$ & \\
\hline Oxygen saturation & $9(14 \%)$ & $3(19 \%)$ & $6(12 \%)$ & \\
\hline Respiratory rate & $2(3 \%)$ & $1(6 \%)$ & $1(2 \%)$ & \\
\hline Urine output & $1(2 \%)$ & $1(6 \%)$ & 0 & \\
\hline Other & $11(17 \%)$ & $2(13 \%)$ & $9(18 \%)$ & \\
\hline \multicolumn{5}{|l|}{ Time of dayb } \\
\hline Day (8 a.m. -5 p.m.) & $32(49 \%)$ & $6(38 \%)$ & $26(53 \%)$ & \\
\hline Night (5 p.m.-8 a.m.) & $33(51 \%)$ & $10(63 \%)$ & $23(47 \%)$ & \\
\hline
\end{tabular}

Values other than $P$ value and number of patients are presented as median (range) or as number (percentage). $P$ value represents the probability of an error when concluding that the characteristic differs by adverse event (AE) status. aLength of stay in hospital before Medical Emergency Team (MET) call; btime of day of MET call. N/A, not applicable.

\section{Discussion}

We found our MET-based approach for preventable adverse event detection to be simple to implement, easy to maintain, and informative for quality improvement efforts. One quarter of MET calls were associated with preventable adverse events. We found one service responsible for a disproportionate number of preventable adverse events. We also found inappropriate responses to critical patients as the most common cause of preventable adverse events. Our hospital is using this information to guide quality improvement strategies.

Our program cost very little to implement. Although we collected data specifically for the study, it is possible for the care providers present at the MET call to incorporate information collected at each call directly into the routine. The program required weekly meetings, which lasted less than an hour and could be performed remotely using telephone conferencing. This task was not onerous for the physicians participating in the program and was seen as part of their professional obligations of monitoring the effectiveness of the hospital system.

Although we believe our methodology is easily replicable, our surveillance results should not be generalized. Our study was performed in a single site for a limited period of time. Despite the relatively short observation period, we did identify a statistically significant and clinically plausible pattern of factors associated with preventable adverse events. Prior research has suggested that, even in acute care hospitals, there is often an inadequate response to critically ill patients [12-16]. Furthermore, a prior program similar to ours, but which observed 
care for 8 months, found a similar proportion of MET calls to be related to preventable adverse events [17]. In the prior study, the predominant problem was diagnostic error. It is possible that, if the observation period of our study had been longer, we would have found different patterns. It is also possible that our studies used slightly different terminologies to classify the type of adverse events. Thus, despite the consistency with prior research, we recommend a larger study. Such a study should ensure standard terminology and consider comparing the preventable adverse events detected by this method with those identified using other methods to ensure validation of the types of preventable adverse events occurring in an institution.

Similarly, it is important to consider specific biases inherent in this approach to finding care-related problems in a hospital. The physician review process is biased by knowledge of outcome severity and by our natural and variable inclinations to find fault $[25,26]$. These biases can be minimized by having multiple reviewers [27] and by blinding outcome severity [25]. However, the impact of these biases can be mitigated but not entirely removed. As a result, any findings from an MET-based surveillance program should be interpreted and communicated cautiously. We suggest that they function as a starting point for assessments that are more intensive rather than as the basis of sanctions. Furthermore, we strongly suggest adopting a communication strategy that avoids blaming individuals or groups for negligence or incompetence. Rather, the findings should be used in a constructive and collaborative manner to plan future assessments and quality improvement efforts.

\section{Conclusions}

Given the widespread implementation of METs, our proposed approach could immediately offer many hospitals an efficient method for monitoring preventable adverse events. This is an important advance given the apparent widespread patient safety problems in hospitals $[19,20,28-30]$ and the inadequacy of existing surveillance systems [31-33].

\section{Competing interests}

The authors declare that they have no competing interests.

\section{Authors' contributions}

AJF conceived of the idea of the study and helped to facilitate data collection and provide important intellectual contributions during preparation of the manuscript. $A I$ and $A B$ helped to facilitate data collection and provide important intellectual contributions during preparation of the manuscript. All authors read and approved the final manuscript.

\section{Key messages}

- Medical emergency teams (METs) provide care to critically ill ward-based inpatients. METs have been implemented in many hospitals worldwide.

- METs often respond to clinical events in which there has been inappropriate antecedent care. Therefore, METs could form the basis of a preventable adverse event detection system.

- We have adopted a structured method of data collection and peer review to be used by METs to assist institutional learning regarding the avoidance of preventable adverse events.

- We have determined our method to be feasible.

- We have demonstrated the method's capacity to document important quality issues in the care of critically ill patients.

\section{Additional files}

The following Additional files are available online:

\section{Additional file 1}

Appendix 1 containing our case review form. See http://www.biomedcentral.com/content/ supplementary/cc7983-S1.DOC

\section{Additional file 2}

A text box with several examples of adverse events identified during the study.

See http://www.biomedcentral.com/content/ supplementary/cc7983-S2.DOC

\section{Additional file 3}

Appendix 2 with descriptions of all adverse events identified during the study.

See http://www.biomedcentral.com/content/ supplementary/cc7983-S3.DOC

\section{Acknowledgements}

AJF is supported by an Ontario Ministry of Health Career Scientist Award. This research received funding from the Canadian Patient Safety Institute, the Canadian Institute for Health Research, the Healthcare Insurance Reciprocal of Canada, the University of Ottawa Heart Institute, and the Ottawa Hospital Center for Patient Safety.

\section{References}

1. Berwick DM, Calkins DR, McCannon CJ, Hackbarth AD: The 100,000 Lives Campaign: setting a goal and a deadline for improving health care quality. JAMA 2006, 295:324-327. 
2. Bellomo R, Goldsmith D, Uchino S, Buckmaster J, Hart GK, Opdam H, Silvester W, Doolan L, Gutteridge G: A prospective before-and-after trial of a medical emergency team. Med $J$ Aust 2003, 179:283-287.

3. Bellomo R, Goldsmith D, Uchino S, Buckmaster J, Hart G, Opdam $H$, Silvester W, Doolan L, Gutteridge G: Prospective controlled trial of effect of medical emergency team on postoperative morbidity and mortality rates. Crit Care Med 2004, 32:916-921.

4. Buist MD, Moore GE, Bernard SA, Waxman BP, Anderson JN, Nguyen TV: Effects of a medical emergency team on reduction of incidence of and mortality from unexpected cardiac arrests in hospital: preliminary study. BMJ 2002, 324:387-390.

5. DeVita MA, Braithwaite RS, Mahidhara R, Stuart S, Foraida M, Simmons RL: Use of medical emergency team responses to reduce hospital cardiopulmonary arrests. Qual Saf Health Care 2004, 13:251-254.

6. Hillman K, Chen J, Cretikos M, Bellomo R, Brown D, Doig G, Finfer $S$, Flabouris A, MERIT study investigators: Introduction of the medical emergency team (MET) system: a cluster-randomised controlled trial. Lancet 2005, 365:2091-2097.

7. Kenward G, Castle N, Hodgetts T, Shaikh L: Evaluation of a medical emergency team one year after implementation. Resuscitation 2004, 61:257-263.

8. Chan PS, Khalid A, Longmore LS, Berg RA, Kosiborod M, Spertus JA: Hospital-wide code rates and mortality before and after implementation of a rapid response team. JAMA 2008, 300:2506-2513.

9. Lokajner G, Radolic D, Zagar A, Ecimovic P: Introducing rapid response teams in Slovenia. Crit Care 2008, 12:P363.

10. Jones D, George C, Hart GK, Bellomo R, Martin J: Introduction of medical emergency teams in Australia and New Zealand: a multi-centre study. Crit Care 2008, 12:R46.

11. Jones $D$, Bellomo R: Introduction of a rapid response system: why we are glad we MET. Crit Care 2006, 10:121.

12. Buist MD, Jarmolowski E, Burton PR, Bernard SA, Waxman BP, Anderson J: Recognising clinical instability in hospital patients before cardiac arrest or unplanned admission to intensive care. A pilot study in a tertiary-care hospital. Med J Aust 1999, 171:22-25.

13. Hillman KM, Bristow PJ, Chey T, Daffurn K, Jacques T, Norman SL, Bishop GF, Simmons G: Antecedents to hospital deaths. Intern Med J 2001, 31:343-348.

14. Bedell SE, Deitz DC, Leeman D, Delbanco TL: Incidence and characteristics of preventable iatrogenic cardiac arrests. JAMA 1991, 265:2815-2820.

15. Smith AF, Wood J: Can some in-hospital cardio-respiratory arrests be prevented? A prospective survey. Resuscitation 1998, 37:133-137.

16. Kause J, Smith G, Prytherch D, Parr M, Flabouris A, Hillman K: A comparison of antecedents to cardiac arrests, deaths and emergency intensive care admissions in Australia and New Zealand, and the United Kingdom - the ACADEMIA Study. Resuscitation 2004, 62:275-282.

17. Braithwaite RS, DeVita MA, Mahidhara R, Simmons RL, Stuart S, Foraida M: Use of medical emergency team (MET) responses to detect medical errors. Qual Saf Health Care 2004, 13:255-259.

18. Institute of Medicine: To Err Is Human: Building a Safer Health System Edited by: Kohn LT, Corrigan JM, Donaldson MS. Washington, DC: National Academies Press; 2000.

19. Brennan TA, Leape LL, Laird NM, Hebert L, Localio AR, Lawthers AG, Newhouse JP, Weiler PC, Hiatt HH: Incidence of adverse events and negligence in hospitalized patients. Results of the Harvard Medical Practice Study I. N Engl J Med 1991, 324:370-376.

20. Wilson RM, Runciman WB, Gibberd RW, Harrison BT, Newby L, Hamilton JD: The Quality in Australian Health Care Study. Med $J$ Aust 1995, 163:458-471.

21. Rothschild JM, Landrigan CP, Cronin JW, Kaushal R, Lockley SW, Burdick E, Stone PH, Lilly CM, Katz JT, Czeisler CA, Bates DW: The Critical Care Safety Study: the incidence and nature of adverse events and serious medical errors in intensive care. Crit Care Med 2005, 33:1694-1700.

22. Bates DW, Leape LL, Petrycki S: Incidence and preventability of adverse drug events in hospitalized adults. J Gen Intern Med 1993, 8:289-294.
23. Forster AJ, Clark HD, Menard A, Dupuis N, Chernish R, Chandok $\mathrm{N}$, Khan $\mathrm{A}$, van Walraven C: Adverse events affecting medical patients following discharge from hospital. CMAJ 2004, 170:345-349.

24. Forster AJ, Murff HJ, Peterson JF, Gandhi TK, Bates DW: The incidence and severity of adverse events affecting patients after discharge from the hospital. Ann Intern Med 2003, 138:161-167.

25. Caplan RA, Posner KL, Cheney FW: Effect of outcome on physician judgments of appropriateness of care. JAMA 1991, 265:1957-1960.

26. Brennan TA, Localio RJ, Laird NL: Reliability and validity of judgments concerning adverse events suffered by hospitalized patients. Med Care 1989, 27:1148-1158.

27. Forster AJ, O'Rourke K, Shojania KG, van Walraven C: Combining ratings from multiple physician reviewers helped to overcome the uncertainty associated with adverse event classification. $J$ Clin Epidemiol 2007, 60:892-901.

28. Vincent C, Neale G, Woloshynowych M: Adverse events in British hospitals: preliminary retrospective record review. $B M J$ 2001, 322:517-519.

29. Thomas EJ, Studdert DM, Burstin HR, Orav EJ, Zeena T, Williams EJ, Howard KM, Weiler PC, Brennan TA: Incidence and types of adverse events and negligent care in Utah and Colorado. Med Care 2000, 38:261-271.

30. Baker GR, Norton PG, Flintoft V, Blais R, Brown A, Cox J, Etchells E, Ghali WA, Hébert P, Majumdar SR, O'Beirne M, Palacios-Derflingher L, Reid RJ, Sheps S, Tamblyn R: The Canadian Adverse Events Study: the incidence of adverse events among hospital patients in Canada. CMAJ 2004, 170:1678-1686.

31. Pronovost PJ, Miller MR, Wachter RM: Tracking progress in patient safety: an elusive target. JAMA 2006, 296:696-699.

32. Gawande A, Thomas E, Studdert D: Accidental deaths, saved lives, and improved quality. N Engl J Med 2005, 353:1405-1409.

33. Thomas EJ, Petersen LA: Measuring errors and adverse events in health care. J Gen Intern Med 2003, 18:61-67. 